\title{
A Bespoke Single-Band Hubbard Model Material
}

\author{
S. M. Griffin, ${ }^{1}$ P. Staar, ${ }^{2}$ T. C. Schulthess,${ }^{2,3}$ M. Troyer,${ }^{2}$ and N. A. Spaldin ${ }^{1, *}$ \\ ${ }^{1}$ Materials Theory, ETH Zurich, Wolfgang-Pauli-Strasse 29, CH-8093 Zürich, Switzerland \\ ${ }^{2}$ Institute for Theoretical Physics, ETH Zurich, CH-8093 Zürich, Switzerland \\ ${ }^{3}$ Swiss National Supercomputing Center, ETH Zurich, CH-6900 Lugano, Switzerland
}

\begin{abstract}
The Hubbard model, which augments independent-electron band theory with a single parameter to describe electron-electron correlations, is widely regarded to be the 'standard model' of condensed matter physics. The model has been remarkably successful at addressing a range of correlation effects in solids, but beyond one dimension its solution is intractable. Much current research aims, therefore, at finding appropriate approximations to the Hubbard model phase diagram. Here we take the new approach of using ab initio electronic structure methods to design a material whose Hamiltonian is that of the single-band Hubbard model. Solution of the Hubbard model will then be available through measurement of the material's properties. After identifying an appropriate crystal class and several appropriate chemistries, we use density functional theory and dynamical mean-field theory to screen for the desired electronic band structure and metal-insulator transition. We then explore the most promising candidates for structural stability and suitability for doping and propose specific materials for subsequent synthesis. Finally, we identify a regime - that should manifest in our bespoke material - in which the single-band Hubbard model on a triangular lattice exhibits exotic $d$-wave superconductivity.
\end{abstract}

\section{INTRODUCTION}

Independent-electron band theory - in which interactions between electrons are treated at a mean-field level is a simple yet often remarkably effective method for calculating the behavior of electrons in solids. In its modern density functional implementation, its combination of explicit description of crystal structure and chemistry with computational affordability has led to many successes ranging from early predictions of phase stability and lattice dynamics in technologically important materials such as semiconducting silicon ${ }^{1}$, through description of the structure and electronics of lattice defects ${ }^{2}$, to modern predictions of new physics and phenomena in complex materials such as nano- and heterostructures ${ }^{3}$.

By construction, however, independent-electron theories do not include explicit correlations between individual electrons and so are often inappropriate for describing or predicting the properties of so-called stronglycorrelated materials, in which these interactions dominate the physics. Its classic failure is the inability to predict an insulating state for a half-filled band, which will always be metallic within the conventional band theory formalism. Here model Hamiltonians with explicit electon-electron interaction terms have an advantage, at the expense of loss of computational tractability and chemical information. In particular, Hubbard introduced the modern form of the model that now bears his name in a series of papers beginning in $1963^{4-6}$ specifically to "set up the simplest possible model containing the necessary ingredients". (We note that the first hints of the Hubbard model can be found in the quantum chemistry literature in the $1950 \mathrm{~s}^{7-9}$, with P. W. Anderson also suggesting an early variation ${ }^{10}$, and the standard form also independently proposed by Gutzwiller ${ }^{11}$ and Kanamori $\left.{ }^{12}\right)$. The
Hubbard Hamiltonian is

$$
H=-t \sum_{\langle i, j\rangle \sigma}\left(c_{i, \sigma}^{\dagger} c_{j, \sigma}+c_{j, \sigma}^{\dagger} c_{i, \sigma}\right)+U \sum_{i=1}^{N} n_{i \uparrow} n_{i \downarrow} .
$$

Here the first term is the kinetic energy term of standard band theory, with $\langle i, j\rangle$ indicating summation over all pairs of lattice sites (usually nearest neighbours), $\sigma$ indicating the spin degree of freedom, and $t$ the hopping matrix element between states $i$ and $j . c_{i}^{\dagger}$ and $c_{i}$ are the usual creation and annihilation operators, and the number operator, $n_{i}=c_{i}^{\dagger} c_{i}$. The second term introduces explicit electron-electron correlations through the Coulomb repulsion, of magnitude $U$, between two electrons occupying the same lattice site. We illustrate both terms schematically in Fig. 1.

For small values of $U$ and large values of $t$ (the weakcorrelation limit), Eqn. 1 yields metallic solutions for partially filled bands and can successfully describe, for example, itinerant magnetism in transition metals. Importantly, increasing the ratio of $U / t$ (the strong-correlation limit) causes a crossover to an insulating solution, allowing the description of, for example, the antiferromagnetic insulating state of transition metal oxides. Modern studies can incorporate (non-self-consistent) information about crystal structure and chemistry via density functional calculations of the $t$ and $U$ parameters, and an increasing range of materials from Mott insulators to high- $T_{c}$ superconductors is currently addressed ${ }^{13-16}$. While it is not obvious why a single-band picture is relevant in the case of systems with degenerate $d$ orbitals or strong hybridization with $s$ or $p$ states, several works show that the single-band picture does indeed successfully describe low-energy charge and spin excitations in a realistic manner ${ }^{17,18}$.

Despite the simplicity of Eqn. 1, the general Hubbard Model is in fact computationally intractable, and much 
current research activity aims at obtaining the Hubbard model phase diagram. Exact solutions are possible in one dimension ${ }^{19}$, where a transition from a metal to a Mott insulator occurs at half filling when $U>t^{20}$. Away from exactly half filling the solution is always metallic for either hole or electron doping. In two dimensions, numerically exact solutions can be found in the ground state only for either very small lattices of at most about twenty sites ${ }^{21}$, or on so-called ladder models consisting of coupled chains for around 100 sites using the density matrix renormalization group algorithm ${ }^{22}$. For arbitrary dimension and large lattices, however, approximations must be used to investigate the phase diagram. Analytical methods such as slave-boson mean field theory and the Gutzwiller approximation have been applied extensively ${ }^{23-25}$, and numerical techniques such as the Dynamical Cluster Approximation (DCA) and Dynamical Mean-Field Theory (DMFT) have had some success ${ }^{14,26-28}$. The rapid scaling of the computational resources required with the system size has hampered efforts in simulating large systems, however. Despite this profusion of computational approaches, consensus has yet to be reached on the exact nature of the phase diagram throughout the entire phase space, although diverse numerical methods start to agree on some properties of the 2D Hubbard model ${ }^{29,30}$. Much less is known in three dimensions, where substantial interest has been raised by the possibility of realizing the Hubbard model in optical lattices ${ }^{31-33}$ and simulations have mainly been performed by quantum Monte Carlo simulations at relatively high temperatures of the order of the Néel temperature ${ }^{34-39}$.

In this work we take a new approach to "solving" the single-band Hubbard model and use ab initio electronic structure methods, at the band theory and dynamical mean field theory levels, to design a real material whose Hamiltonian is exactly that of the singleband Hubbard model. Measurement of the properties of our bespoke material would then allow extraction of the Hubbard model phase diagram. Our approach is somewhat reminiscent of experimental simulations of the Hubbard model that have been performed using ultracold atoms ${ }^{40}$, which take the role of the the electrons in the conventional Hubbard model. The atoms are trapped in a potential created by interfering laser beams and since the lasers are highly controllable, a vast range of potentials can be created using different interference effects. The simulated lattice model is therefore highly tunable both in terms of the lattice geometry and dimensionality, and in the value of $t / U$. In particular, a metal-insulator transition, accompanied by a suppression of doubly-occupied sites, a drop in compressibility and an excitation gap, has been demonstrated in a gas of cold fermionic atoms by tuning the strength of their mutual repulsion ${ }^{41,42}$, and short range magnetic correlations have been observed ${ }^{43,44}$. While proposals have been made to cool below the Néel temperature, however, (see, for example Ref. 37) to achieve long range magnetic oder and adiabatically prepare $d$-wave superconducting phases $^{45}$, experimental progress has been slow and challenging. A condensed matter realization of the Hubbard model, even if not as tunable as ultracold quantum gases, will thus be interesting as it will allow much lower temperatures to be reached.

\section{DESIGN OF A SINGLE-BAND HUBBARD MODEL MATERIAL}

\section{A. Candidate crystal structures and chemistries}

Since we seek a material described by a singleband Hubbard model, we begin by engineering a nondegenerate $d$-manifold using crystal-field considerations. Crystal-field splittings with single non-degenerate $d$ bands are obtained for a variety of ionic geometries, such as pentagonal bipyramidal, square antiprismatic, square planar, square pyramidal and trigonal bipyramidal (Fig. 2). In this work we choose the trigonal bipyramidal coordination, in which the five-fold degeneracy is broken into two doubly-degenerate orbital levels $\left(d_{x z}, d_{y x}\right.$ and $\left.d_{x y}, d_{x^{2}-y^{2}}\right)$, and a single orbital $\left(d_{z^{2}}\right)$, for its combination of large energetic separation of the single-band state and geometric simplicity. For trigonal bipyramidal coordination with shorter apical than in-plane ligand distances, the isolated $d_{z}^{2}$ band is the highest energy level, and so it becomes half filled for a $d^{9}$ electron configuration.

Such trigonal bipyramidal coordination is found in the well-studied hexagonal manganite structure adopted by $\mathrm{YMnO}_{3}$ as well as the manganites of the smaller rare earth ions. The structure is formed from layers of corner-sharing $\mathrm{MnO}_{5}$ trigonal bipyramids alternating with planes of $\mathrm{Y}$ ions. Figs. 3(a) and (b) show the highsymmetry $P 6_{3} / m m c$ variant that occurs at high temperature; below around $1000 \mathrm{~K}$, there is a phase transition to a ferroelectric $P 6_{3} \mathrm{~cm}$ structure, which does not alter the coordination environment and hence does not change the crystal-field splitting. In Fig. 3(c) we show our calculated density of states for hexagonal $\mathrm{YMnO}_{3}$ in the high symmetry $P 6_{3} / m m c$ reference structure, and with the local moments on the Mn ions ferromagnetically aligned. The $3 d^{4} \mathrm{Mn}^{3+}$ ions have a high-spin configuration, with the up-spin $d_{x z}, d_{y x}$ and $d_{x y}$ and $d_{x^{2}-y^{2}}$ orbitals occupied, and the spin-up $d_{z^{2}}$ forming the lowest energy state above the Fermi level. The exchange-split spin-down states begin at an energy of $\sim 4 \mathrm{eV}$ above the Fermi level. While the $d^{4} \mathrm{Mn}^{3+}$ configuration is magnetic and does not yield a half-filled single band, excluding it from consideration as our single-band Hubbard material, we see already that the $d_{z}^{2}$ orbitals form an isolated band with little hybridization with the oxygen ligands, confirming our intuition that this crystal class is promising.

Next we select ion combinations that have formal valence states that should yield half-filling of the isolated $d_{z}^{2}$ band, and will also likely allow for doping across the range from empty to filled band. As mentioned above, 
to half-fill the $d_{z^{2}}$ band, our B-site cation should have a $d^{9}$ configuration, suggesting $\mathrm{Cu}^{2+}$ as the most promising candidate. For oxides or sulphides, both of which have divalent anions, the A-site cations should be four valent suggesting $\mathrm{Zr}$ or $\mathrm{Sn}$ as possibilities. For fluorides, in which the anions are monovalent, the A-sites should also be monovalent, suggesting $\mathrm{Li}$ or Na. We take, therefore the following as our initial list of trial compounds for further study: $\mathrm{ZrCuO}_{3}, \mathrm{SnCuO}_{3} ; \mathrm{ZrCuS}_{3}, \mathrm{SnCuS}_{3}$; $\mathrm{LiCuF}_{3}, \mathrm{NaCuF}_{3}$.

\section{B. Computational details}

Our electronic structure calculations were performed within density-Functional theory using the Vienna $a b$ initio Simulation Package (VASP) ${ }^{46,47}$. The electronic wavefunctions and density were expanded using a planewave basis set, and we used projector-augmented-wave (PAW) potentials ${ }^{48}$ for core-valence separation. For the exchange-correlation potential we used the generalized gradient approximation (GGA) ${ }^{49,50}$ and strong correlation effects were treated by means of the $G G A+U$ scheme. Here we used the Dudarev method ${ }^{51}$ in which a $U_{\text {eff }}=U-J$ accounts for the on-site $U$ (Coulomb replusion) and $J$ (Hund's rule exchange) on the metal $d$ states. To choose an appropriate $U_{\text {eff }}$ for hypothetical compounds (without spectroscopic data), we performed hybrid functional calculations to select an appropriate range by matching the DFT $+U$ band gap with the hybrid functional result. In the hybrid functional calculations, an HSE functional consisting of $75 \% \mathrm{PBE}$ and $25 \%$ exact Hartree-Fock exchange was used ${ }^{52}$. For our Cu-based compounds, this led us to choose a $U_{\text {eff }}$ of $7 \mathrm{eV}$ on the $\mathrm{Cu}-d$ states, which is similar to that used in previous studies on cuprate materials ${ }^{53}$. (The calculated $\mathrm{YMnO}_{3}$ DOS shown in Fig. 3 (c) was also obtained using a $U_{\text {eff }}$ of $7 \mathrm{eV}$ on the Mn- $d$ states, consistent with literature studies on $\mathrm{YMnO}_{3}$.) A $10 \times 10 \times 4$ Gamma-centred kpoint mesh was used for Brillouin-zone integrations. The plane-wave cut-off was set to $550 \mathrm{eV}$ and in performing the structural optimizations, we allowed the ions to relax until the Hellmann-Feynman forces were less than 1 $\mathrm{meV} / \AA^{-1}$.

To treat the electron-electron correlations in modeling the metal-insulator transition, we used the Dynamical Mean-Field Theory (DMFT) approach ${ }^{54}$. We first constructed maximally localized Wanner functions for the transition-metal $d$ bands of the Kohn-Sham Hamiltonian using the wannier90 $\operatorname{code}^{55,56}$. We then used these as the noninteracting part $H_{0}$ of a Hubbard Hamiltonian $H=H_{0}+H_{\text {int }}$ where $H_{\text {int }}$ is the local electron-electron interaction comprising the intra-orbital Coulomb interaction, $U_{D M F T}$, and Hund's rule coupling, $J$. Here we used $J=0 \mathrm{eV}$ since we consider a single orbital, while we varied $U_{D M F T}$ from 0 to $4 \mathrm{eV}$. We used a continuous-time hybridization expansion quantum Monte Carlo solver ${ }^{57}$ as implemented in the TRIQS $1.0 \operatorname{code}^{58}$ to calculate the local Green's function within DMFT at a temperature $T=1 /\left(k_{B} \beta\right)=290 \mathrm{~K}\left(\beta=40 \mathrm{eV}^{-1}\right)$.

\section{RESULTS}

\section{A. Structural optimization}

First we perform an initial band structure screening of our trial compounds within the high-temperature $\mathrm{P6}_{3} / \mathrm{mmc}$ structure. Our calculated lattice constants for all six compounds are given in Table I. For the oxides and fluorides, the transition metal - apical ligand distances are shorter than the corresponding in-plane distances, which should yield the highest energy singly degenerate crystal field state required in our analysis. As expected from the larger atomic radius of $\mathrm{Na}$, the volume of $\mathrm{NaCuF}_{3}$ is slightly greater than that of $\mathrm{LiCuF}_{3}$, although the apical $\mathrm{Cu}-\mathrm{F}$ bonding distances are almost the same in both compounds. Between the $\mathrm{Zr}$ and $\mathrm{Sn}$ oxides, the volumes and $\mathrm{Cu}-\mathrm{O}$ distances are almost identical, suggesting similar crystal-field splittings in the two cases. Interestingly, for the sulphides, the apical $\mathrm{Cu}-\mathrm{S}$ bonding distances are longer than the in-plane distances. This should place the singly degenerate $d_{z}^{2}$ band in the lowest energy position which will lead to its full application. Therefore we exclude the sulphides from our list of candidates for further consideration.

\section{B. Electronic properties at the density functional theory level}

Using our optimized structures, we next calculate the electronic properties of the candidate materials at the density functional theory level. Fig. 4 shows the calculated electronic band structures and orbital-resolved densities of states for (a) $\mathrm{ZrCuO}_{3}$, (b) $\mathrm{SnCuO}_{3}$, (c) $\mathrm{LiCuF}_{3}$ and (d) $\mathrm{NaCuF}_{3}$. For both of the oxide compounds, the Fermi energy intersects a band that is composed primarily of $\mathrm{Cu}-d_{z^{2}}$ states, with some admixture from apical $\mathrm{O}-p_{z}$ states. The $\mathrm{Cu}-d_{z^{2}}$ band is almost separated from the broad valence band composed of the other four majority $\mathrm{Cu}-d$ bands and oxygen, but the separation is not complete due to its non-negligible bandwidth of $1.2 \mathrm{eV}$. For the tin case, we also find Sn- $s$ states at $\mathrm{E}_{F}$ (Fig. 4 (b)), which are undesirable for achieving a single-band model.

We find a more promising situation in the fluorides (Figs. 4 (c) and (d)) both of which show a completely split-off half-filled $\mathrm{Cu}-d_{z^{2}}$ band. The reduced anion hybridization in these more ionic compounds gives a smaller bandwidth of $\sim 1 \mathrm{eV}$ and makes the fluorides more singleband-like. (Note that our calculation is for a twoformula-unit unit cell, and the two $\mathrm{Cu}$ ions are slightly symmetry inequivalent so two $\mathrm{Cu} d_{z}^{2}$ bands are shown.) The electronic structures of $\mathrm{LiCuF}_{3}$ and $\mathrm{NaCuF}_{3}$ are remarkably similar in the region surrounding the Fermi 
level since there is no contribution from the A sites in either case in this region. The only clear difference is that the crystal-field gap between the split-off band and the other $\mathrm{Cu}-d$ states is slightly larger in $\mathrm{NaCuF}_{3}(\sim$ $0.3 \mathrm{eV})$ than in $\mathrm{LiCuF}_{3}(\sim 0.2 \mathrm{eV})$.

In summary, we find from density functional calculations that the cuprates of $\mathrm{Zn}$ and $\mathrm{Sn}$, and the copper fluorides of $\mathrm{Li}$ and $\mathrm{Na}$ are promising candidate singleband Hubbard model materials when in the hexagonal manganite structure, with the fluorides having the more desirable properties. Next we continue with one of the fluoride representatives - $\mathrm{LiCuF}_{3}$ - and investigate the effect of explicit electron correlations at the dynamical mean field theory level.

\section{Electronic structure at the dynamical mean field theory level}

To study the effect of explicit electron-electron correlations on the $\mathrm{d}_{z}^{2}$ band, we next calculate the spectrum of $\mathrm{LiCuF}_{3}$ using dynamical mean field theory (DMFT). We first construct the maximally-localized Wannier function (Fig. 5 (a)) on a $\mathrm{Cu}$ site for the energy window corresponding to the isolated $\mathrm{Cu}-d_{z^{2}}$ band using the Wannier90 $\operatorname{code}^{56}$. As expected the Wannier function has primarily $d_{z^{2}}$ character with small $p$-symmetry "tails" extending onto the neighboring fluorine ions.

Using this Wannier function as a basis, we next calculate the spectral function, $A(\omega)$ within DMFT, by analytic continuation using the maximum entropy method ${ }^{59}$ Our results are shown in Fig. 5(b) for $U_{D M F T}$ values ranging from 0 to $1.5 \mathrm{eV}$. We see that the system is metallic at $U=0 \mathrm{eV}$. As the interaction parameter $U$ is increased, the spectral weight gradually shifts from the quasiparticle peak at $\omega=0 \mathrm{eV}$ to the upper and lower Hubbard bands. Eventually a classic Mott transition occurs when the electron interactions are strong enough to cause the quasiparticle peak to disappear completely and a gap to form. Consistent with our $\operatorname{Tr} G(\beta / 2)$ data (not shown), the system gaps above $U_{D M F T}=1.25 \mathrm{eV}$. We comment briefly on the apparent inconsistency introduced by our use of a non-zero $U$ value in our original DFT calculations, combined with a second $U$ in the DMFT study. To address this issue, we repeated our DFT calculations in the GGA $(U=0)$ limit and found the band structure and band widths to be very similar to the $\mathrm{GGA}+U$ case, with the $\mathrm{DFT}+U$ treatment acting as a scissors operator to isolate the $d_{z^{2}}$ orbital. Therefore we expect only small quantitative changes in the $U_{D M F T}$ value at the metal-insulator transition if the initial Wannier functions were constructed from results of the $U=0$ GGA calculation; construction of the Wannier functions would be complicated, however, by the poorly isolated bands.

\section{Structural stability}

As a first step to assessing the feasibility of synthesizing our proposed compounds, we calculate the relative structural stabilities of a range of structural isomorphs of $\mathrm{LiCuF}_{3}$. We consider the following crystal structures all of which are known to exist for compounds with $A B X_{3}$ chemistry: high-temperature hexagonal manganite $\left(P 6_{3} / m m c\right)$, low-temperature ferroelectric hexagonal manganite $\left(\mathrm{P}_{3} \mathrm{~cm}\right)$, low-temperature nonpolar hexagonal manganite $(P \overline{3} c)$, cubic perovskite $(P m \overline{3} m)$, ilmenite $(R \overline{3})$, the ' $\mathrm{NaCuF}_{3}$ ' structure (which corresponds to the ambient experimental ground state, $P \overline{1})$, the ' $\mathrm{CoGeO}_{3}$ ' structure $(C 2 / c)$, and two non-centrosymmetric structural variants - rhombohedral $(R 3 m)$ and tetragonal $(P 4 / m m m)$ - found in $\mathrm{BaTiO}_{3}$. We apply both positive and negative hydrostatic pressure by scaling the lattice parameters, keeping the fractional internal coordinates of the ions fixed for each calculation to ensure that the original symmetry group is maintained.

Fig. 6 shows the resulting calculated energies as a function of volume for the various structural isomorphs of $\mathrm{LiCuF}_{3}$ that were lowest in energy over the range of volumes studied. We find that the lowest energy structure is the ' $\mathrm{NaCuF}_{3}$ ' structure, however for an $8 \%$ increase in volume (corresponding to a $2 \%$ increase in lattice constant) the hexagonal manganite structure is the ground state, suggesting that it might be possible to achieve it using tensile strain. At much larger lattice constants the $C 2 / c \mathrm{CoGeO}_{3}$ structure becomes stable.

\section{E. Doping}

Finally, we explore routes to electron- and hole- doping $\mathrm{LiCuF}_{3}$ so that the entire range of band filling can be accessed. First we artificially add (remove) up to one electron per formula unit without changing the ion configuration, using a compensating background positive (negative) charge to prevent electrostatic divergence. As expected, the Fermi level shifts downwards (upwards) as electrons are removed (added), giving a completely unfilled $\mathrm{Cu}-d_{z^{2}}$ band when $1 \mathrm{e}^{-}$per formula unit is removed, and a completely filled $\mathrm{Cu}-d_{z^{2}}$ band when $1 \mathrm{e}^{-}$per formula unit is added. No qualitative change apart from the rigid shift in the Fermi level is found over the entire doping range; in particular the split-off single-band character is retained.

An experimentally accessible possibility for hole doping is to substitute fluorine (which forms $\mathrm{F}^{-}$, with one negative charge per atom) by oxygen (which forms $\mathrm{O}^{2-}$, with two). Replacing one fluorine atom per unit cell with oxygen yields the chemical formula $\mathrm{Li}_{2} \mathrm{Cu}_{2} \mathrm{~F}_{5} \mathrm{O}_{1}$, which corresponds to removing half an electron per formula unit. We calculated the relative energies of the substitutional sites for the oxygen by replacing each of the two inequivalent fluorine ions with an oxygen ion, performing a full structural relaxation, then comparing 
the final energies. We found that in-plane subsitution is lower in energy by $\sim 0.3 \mathrm{eV}$ per formula unit than apical substitution; this is favorable for our Hubbard material design as the in-plane ions have minimal hybridization with the split-off single band. The resulting density of states (DOS) for in-plane oxygen substitution is plotted in Fig. 7(b) (left panel), with the orbital projected O-p states shown in pink. Note that the electronic structure in the region of the Fermi level is remarkably similar to that obtained by removing electrons in our first doping calculations.

Possible routes to electron doping $\mathrm{LiCuF}_{3}$ are to replace some $\mathrm{Li}$ with $\mathrm{Be}$ or to introduce $\mathrm{F}$ vacancies. Substituting one $\mathrm{Li}$ ion with a Be ion introduces half an extra electron per formula unit with the chemical formula $\mathrm{LiBeCu}_{2} \mathrm{~F}_{6}$. The influence of Be substitution on the electronic structure is shown in Fig. 7(b) (right panel), where we have plotted the $\mathrm{Cu}-d_{z^{2}}$ band separately from the other $\mathrm{Cu}-d$ states. While no Be states appear near the Fermi level, the substitution causes an orbital reordering whereby the region next to the Fermi level now comprises contributions from the full $d$ manifold. Removing one fluorine atom per unit cell to give the chemical formula $\mathrm{Li}_{2} \mathrm{Cu}_{2} \mathrm{~F}_{5}$ also corresponds to adding half an electron per formula unit. We calculated the relative energy of vacancy sites by removing the fluorine ion from each of the two inequivalent fluorine in turn and performing a full internal relaxation. We found that apical fluorine vacancies are lower in energy than in-plane vacancies by $\sim 0.1 \mathrm{eV}$ per formula unit; the resulting density of states for apical vacancies is plotted in Fig. 7(e) (right panel). Unlike the Be substitution case, the region surrounding the Fermi level retains its $\mathrm{Cu}-d_{z^{2}}$ character and behaves more like the rigid-band model of electron addition.

\section{F. Exotic superconductivity}

Since their discovery in the 1980 's ${ }^{60}$, the cuprate superconductors have remained one of the most studied classes of materials, as well as one of the most elusive in revealing a comprehensive understanding of their behavior ${ }^{61,62}$. In particular, while they have the highest critical temperatures of all known superconductors, the details of the pairing mechanism are still unknown. The relevant features for high- $T_{c}$ superconductivity in the cuprates appear to be the quasi two-dimensional electronic structure, the parent antiferromagnetic compound with a range of accessible dopings, the spin- $\frac{1}{2}$ magnetic moment and the single band at the Fermi level. For example, the $\mathrm{La}_{2} \mathrm{CuO}_{4}$ cuprate parent compound contains $3 d^{9}$ octahedrally coordinated $\mathrm{Cu}^{2+}$ ions. A strong Jahn-Teller distortion lifts the energy of the two $e_{g}$ orbitals yielding a non-degenerate half-filled $\mathrm{Cu}-d_{x^{2}-y^{2}}$ band which is, however, strongly hybridized with the $p_{x}$ and $p_{y}$ states on the neighboring oxygens ${ }^{17}$. The ground state is antiferromagnetic and insulating due to the combined effects of exchange splitting and Mott electron repulsion.
Recognition of these characteristics have led to attempts to design new materials that might enhance exotic superconductivity. A particularly clever suggestion was to use geometric engineering to crystal-field split the degenerate $e_{g}$ orbitals rather than relying on the Jahn-Teller distortion $^{63}$. A proposed candidate system was a layered superlattice of $\left(\mathrm{LaNiO}_{3} / \mathrm{LaAlO}_{3}\right)$ in which the symmetry and strain of the superlattice would lift the degeneracy of the Ni $e_{g}$ states. While an intriguing idea, in experiment charge-transfer physics has been found to dominate the behavior and supress superconductivity.

Our bespoke single-band Hubbard material, $\mathrm{LiCuF}_{3}$, exhibits all the necessary ingredients for exotic superconductivity. In particular, the single band at the Fermi level is strongly two-dimensional, and $\mathrm{Cu}^{2+}$ ions are spin- $\frac{1}{2}$ with antiferromagnetic interactions. The triangular lattice might lead to additional exotic behavior. Therefore, as a final analysis, we investigate whether a superconducting transition might occur in this compound and provide a prediction of its critical temperature. For this we use a variant of the Dynamical Cluster Approximation $(\mathrm{DCA})^{64}$, which is the cluster extension of the (DMFT) $)^{54}$. In this method, the original infinite lattice problem is transformed into solution of a finitesized cluster with periodic boundary conditions, embedded in a self-consistent mean field. This transformation is achieved via a coarse-graining procedure of the Green's function, in which the Brillouin zone is divided into $N c$ patches on which the self-energy is assumed to be constant. The DCA is able to treat all short range correlations between the electrons in the cluster exactly, while long-range correlations outside the cluster are taken into account by the embedding self-consistent mean-field. We recently extended the method ${ }^{65-67}$, to allow determination of the critical temperature for large clusters at a strong interaction strength and to treat continuous lattice self energies and vertex functions in momentum space, giving a more accurate description and allowing a more stringent investigation of the superconducting gap function in momentum space ${ }^{67}$. We use this new DCA+ method here.

DCA has previously been used to investigate the pairing mechanism of the Cwoper pairs in the high- $T_{c}$ cuprates $^{68-74}$. In addition, Chen et al. ${ }^{75}$ recently investigated the superconducting transition on a triangular lattice and discovered two divergent pairing susceptibilities, one with $d_{x y}$ symmetry and one with $d_{x^{2}-y^{2}}$ symmetry, for a cluster of size 6 and $U=8.5 t$. Furthermore, their phase diagram suggests that the critical temperature rises as the doping is decreased, up to a possible $T_{c} \approx 0.06 t$ (with $\mathrm{t}$ being the hopping parameter). This further motivates our study, as our $\mathrm{LiCuF}_{3}$ compound has a half-filled band.

We use the DCA+ method at $U=8 t$ to solve a 16 -site cluster, which has been used in earlier papers investigating the high- $T_{c}$ cuprate superconductors to allow for a direct comparison with these earlier calculations. In Fig. 8, we show our calculated leading eigenvalue $\lambda$ of 
the matrix $\Gamma \chi$. If this leading eigenvalue $\lambda$ crosses 1 , the pairing susceptibility $\chi=\chi_{0} /(1-\Gamma \chi)$ diverges and a superconducting transition occurs. As in Ref. 75 , we find two diverging superconducting modes, one with $d_{x y}$ and one with $d_{x^{2}-y^{2}}$ symmetry. In the inset, we show our finding that the leading eigenvalues are linear on a $\log -\log$ plot, which indicates that the eigenvalue behaves as $\lambda \propto \alpha\left(T-T_{c}\right)^{\gamma}$ and is thus in the mean-field regime. This allows us to extrapolate to lower temperatures and find a $T_{c} \approx 0.08$ by solving the equation $\lambda=1$. In order to understand why the $d_{x y}$ superconducting mode is favoured over the $d_{x^{2}-y^{2}}$, one can look at their corresponding gap functions $\Phi$, defined as the corresponding eigenvector, in momentum space. In Fig. 9 , we show the momentum-space structure of the gap function at the lowest matsubara frequency for respectively the $d_{x y}$ and the $d_{x^{2}-y^{2}}$ superconducting modes as well as a cut of the superconducting gaps along the Fermi surface. We see that the amplitude of the $d_{x y}$ mode is larger than that of the $d_{x^{2}-y^{2}}$ mode, which leads to a larger lambda and critical temperature.

\section{SUMMARY}

We have identified a class of materials with the hexagonal manganite structure whose low-energy behavior reflects the single-band Hubbard Hamiltonian, and propose $\mathrm{LiCuF}_{3}$ and $\mathrm{NaCuF}_{3}$ as promising candidates. We used density functional theory, dynamical mean field theory and the dynamical cluster approximation to characterize $\mathrm{LiCuF}_{3}$ in detail and showed that it displays the expected Mott transition, as well as exotic $d$-wave superconductivity. We hope that our work will motivate experimental synthesis and characterization of this and related compounds ${ }^{76,77}$, as well as exploration of bespoke materials with other model Hamiltonians.
* nicola.spaldin@mat.ethz.ch

1 M. T. Yin and M. L. Cohen, Phys. Rev. B 26, 5668 (1982).

2 C. Freysoldt, B. Grabowski, T. Hickel, J. Neugebauer, G. Kresse, A. Janotti, and C. G. Van de Walle, Rev. Mod. Phys. 86, 253 (2014).

3 J. M. Rondinelli and N. A. Spaldin, Adv. Mater. 23, 3363 (2011).

4 J. Hubbard, Proceedings of the Royal Society of London. Series A. Mathematical and Physical Sciences 276, 238 (1963).

5 J. Hubbard, Proceedings of the Royal Society of London. Series A. Mathematical and Physical Sciences 277, 237 (1964).

6 J. Hubbard, Proceedings of the Royal Society of London. Series A. Mathematical and Physical Sciences 281, 401 (1964).

7 R. Pariser and R. G. Parr, The Journal of Chemical Physics 21, 466 (1953).

8 R. Pariser and R. G. Parr, The Journal of Chemical Physics 21, 767 (1953).

9 J. A. Pople, Trans. Faraday Soc. 49, 1375 (1953).

10 P. W. Anderson, Phys. Rev. 115, 2 (1959).

11 M. C. Gutzwiller, Phys. Rev. Lett. 10, 159 (1963).

12 J. Kanamori, Progress of Theoretical Physics 30, 275 (1963).

13 I. Nekrasov, K. Held, N. Blmer, A. Poteryaev, V. Anisimov, and D. Vollhardt, The European Physical Journal B - Condensed Matter and Complex Systems 18, 55 (2000).

14 K. Held, G. Keller, V. Eyert, D. Vollhardt, and V. I. Anisimov, Phys. Rev. Lett. 86, 5345 (2001).

15 A. I. Lichtenstein and M. I. Katsnelson, Phys. Rev. B 62, R9283 (2000).

16 C. Weber, K. Haule, and G. Kotliar, Nature Physics 6, 574 (2010).

17 F. C. Zhang and T. M. Rice, Phys. Rev. B 37, 3759 (1988).

18 J. Luo and N. E. Bickers, Phys. Rev. B 48, 15983 (1993).

19 F. H. Essler, The one-dimensional Hubbard model (Cambridge University Press, 2005).
${ }^{20}$ E. H. Lieb and F. Wu, Phys. Rev. Lett. 20, 1445 (1968).

${ }^{21}$ S. Yamada, T. Imamura, and M. Machida, in Supercomputing, 2005. Proceedings of the ACM/IEEE SC 2005 Conference (2005) pp. 44-44.

22 R. M. Noack, N. Bulut, D. J. Scalapino, and M. G. Zacher, Phys. Rev. B 56, 7162 (1997).

23 G. Kotliar and A. E. Ruckenstein, Phys. Rev. Lett. 57, 1362 (1986).

${ }^{24}$ H. Hasegawa, Phys. Rev. B 56, 1196 (1997).

25 J. Bünemann and F. Gebhard, Phys. Rev. B 76, 193104 (2007).

${ }^{26}$ T. A. Maier, M. Jarrell, T. Schulthess, P. Kent, and J. White, Physical review letters 95, 237001 (2005).

27 A. Koga, N. Kawakami, T. Rice, and M. Sigrist, Physical review letters 92, 216402 (2004).

28 E. Gull, O. Parcollet, and A. J. Millis, Phys. Rev. Lett. 110, 216405 (2013).

29 D. Scalapino, in Handbook of High-Temperature Superconductivity (Springer, 2007) pp. 495-526.

30 J. P. F. LeBlanc, A. E. Antipov, F. Becca, I. W. Bulik, G. K.-L. Chan, C.-M. Chung, Y. Deng, M. Ferrero, T. M. Henderson, C. A. Jimnez-Hoyos, E. Kozik, X.-W. Liu, A. J. Millis, N. V. Prokof'ev, M. Qin, G. E. Scuseria, H. Shi, B. V. Svistunov, L. F. Tocchio, I. S. Tupitsyn, S. R. White, S. Zhang, B.-X. Zheng, Z. Zhu, and E. Gull, (2015), arXiv:1505.02290.

31 D. Jaksch and P. Zoller, Annals of Physics 315, 52 (2005), special Issue.

32 T. Esslinger, Annu. Rev. Condens. Matter Phys. 1, 129 (2010).

33 M. Lewenstein, A. Sanpera, V. Ahufinger, B. Damski, A. Sen, and U. Sen, Adv Phys 56, 243 (2007).

34 R. Staudt, M. Dzierzawa, and A. Muramatsu, European Physical Journal B 17, 411.

35 P. R. C. Kent, M. Jarrell, T. A. Maier, and T. Pruschke, Phys. Rev. B 72, 060411 (2005).

36 S. Fuchs, E. Gull, L. Pollet, E. Burovski, E. Kozik, T. Pruschke, and M. Troyer, Phys. Rev. Lett. 106, 030401 
(2011)

37 T. Paiva, Y. L. Loh, M. Randeria, R. T. Scalettar, and N. Trivedi, Phys. Rev. Lett. 107, 086401 (2011).

38 E. Kozik, E. Burovski, V. W. Scarola, and M. Troyer, Phys. Rev. B 87, 205102 (2013).

39 J. Imriška, M. Iazzi, L. Wang, E. Gull, D. Greif, T. Uehlinger, G. Jotzu, L. Tarruell, T. Esslinger, and M. Troyer, Physical Review Letters 112, 115301 (2014).

40 M. Greiner, O. Mandel, T. Esslinger, T. W. Hänsch, and I. Bloch, Nature 415, 39 (2002).

41 R. Jördens, N. Strohmaier, K. Günter, H. Moritz, and T. Esslinger, Nature 455, 204 (2008).

${ }^{42}$ U. Schneider, L. Hackermuller, S. Will, T. Best, I. Bloch, T. A. Costi, R. W. Helmes, D. Rasch, and A. Rosch, Science 322, 1520 (2008).

43 D. Greif, T. Uehlinger, G. Jotzu, L. Tarruell, and T. Esslinger, Science 340, 1307 (2013).

44 R. A. Hart, P. M. Duarte, T.-L. Yang, X. Liu, T. Paiva, E. Khatami, R. T. Scalettar, N. Trivedi, D. A. Huse, and R. G. Hulet, Nature 519, 211 (2015).

45 S. Trebst, U. Schollwöck, M. Troyer, and P. Zoller, Physical Review Letters 96, 250402 (2006).

46 G. Kresse and J. Furthmüller, Phys. Rev. B 54, 11169 (1996).

47 G. Kresse and D. Joubert, Phys. Rev. B 59, 1758 (1999).

48 P. E. Blochl, Phys. Rev. B 50, 17953 (1994).

49 J. P. Perdew, K. Burke, and M. Ernzerhof, Phys. Rev. Lett. 77, 3865 (1996).

50 J. P. Perdew, K. Burke, and M. Ernzerhof, Phys. Rev. Lett. 78, 1396 (1997).

51 S. L. Dudarev, G. A. Botton, S. Y. Savrasov, C. J. Humphreys, and A. P. Sutton, Phys. Rev. B 57, 1505 (1998).

52 J. Heyd, G. E. Scuseria, and M. Ernzerhof, The Journal of Chemical Physics 118, 8207 (2003).

${ }^{53}$ I. S. Elfimov, G. A. Sawatzky, and A. Damascelli, Phys. Rev. B 77, 060504 (2008).

54 A. Georges, G. Kotliar, W. Krauth, and M. J. Rozenberg, Reviews of Modern Physics 68, 13 (1996).

55 N. Marzari, A. A. Mostofi, J. R. Yates, I. Souza, and D. Vanderbilt, Rev. Mod. Phys. 84, 1419 (2012).

56 A. A. Mostofi, J. R. Yates, Y.-S. Lee, I. Souza, D. Vanderbilt, and N. Marzari, Computer Physics Communications 178, 685 (2008).

57 E. Gull, A. J. Millis, A. I. Lichtenstein, A. N. Rubtsov, M. Troyer, and P. Werner, Rev. Mod. Phys. 83, 349 (2011).

58 M. Ferrero and O. Parcollet, "TRIQS: A toolbox for research in interacting quantum systems, http://ipht.cea.fr/triqs,".

59 M. Jarrell and J. Gubernatis, Physics Reports 269, 133 (1996).

60 J. G. Bednorz and K. A. Müller, Z. Phys. B 64, 189 (1986).

61 P. Anderson, Princeton Series in Physics (1997).

62 A. J. Leggett, Nature Physics 2, 134 (2006).

63 J. Chaloupka and G. Khaliullin, Phys. Rev. Lett. 100, 016404 (2008).

${ }^{64}$ M. Jarrell, T. Maier, C. Huscroft, and S. Moukouri, Phys. Rev. B 64, 195130 (2001).

65 P. Staar, T. Maier, and T. C. Schulthess, Physical Review B 88, 115101 (2013).

${ }^{66}$ P. Staar, T. A. Maier, R. Solca, G. Fourestey, M. S. Summers, and T. Schulthess, SC - International Conference for High-Performance Computing, Networking, Storage and
Analysis , 11 (2013).

67 P. Staar, T. Maier, and T. C. Schulthess, Physical Review B 89, 195133 (2014).

68 T. A. Maier, M. Jarrell, T. C. Schulthess, P. R. C. Kent, and J. B. White, Phys. Rev. Lett. 95, 237001 (2005).

69 T. A. Maier and D. J. Scalapino, Phys. Rev. B 84, 180513 (2011).

70 S.-Q. Su and T. A. Maier, Phys. Rev. B 84, 220506 (2011).

71 E. Gull, O. Parcollet, and A. J. Millis, Phys. Rev. Lett. 110, 216405 (2013).

72 P. Staar, T. Maier, and T. C. Schulthess, Phys. Rev. B 89, 195133 (2014).

73 E. Gull and A. J. Millis, Phys. Rev. B 90, 041110 (2014).

74 E. Gull and A. J. Millis, Phys. Rev. B 91, 085116 (2015).

75 K. S. Chen, Z. Y. Meng, U. Yu, S. Yang, M. Jarrell, and J. Moreno, Physical Review B 88, 041103 (2013).

76 E. R. Ylvisaker and W. E. Pickett, Phys. Rev. B 74, 075104 (2006).

77 K. W. Lee, J. Kunes, R. T. Scalettar, and W. E. Pickett, Phys. Rev. B 76, 144513 (2007).

\section{ACKNOWLEDGMENTS}

This work was supported by the ETH Zürich, the ERC Advanced Grant program, No. 291151 and the NCCR MARVEL. We thank Gabriele Sclauzero, Claude Ederer, Krzysztof Dymkowski and Sverre M. Selbach for helpful discussions. Calculations were performed at the CSCS Computing Facility.

The authors declare that they have no competing financial interests.

Correspondence and requests for materials should be addressed to nicola.spaldin@mat.ethz.ch 


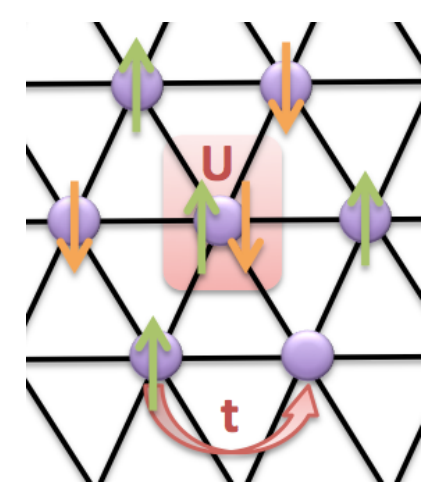

FIG. 1. Cartoon of a triangular lattice illustrating the two parameters that enter the Hubbard model: the inter-site hopping, $t$, and the onsite repulsion, $U$.
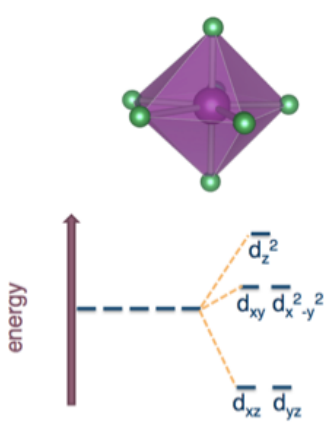

(a) Pentagonal bipyramidal
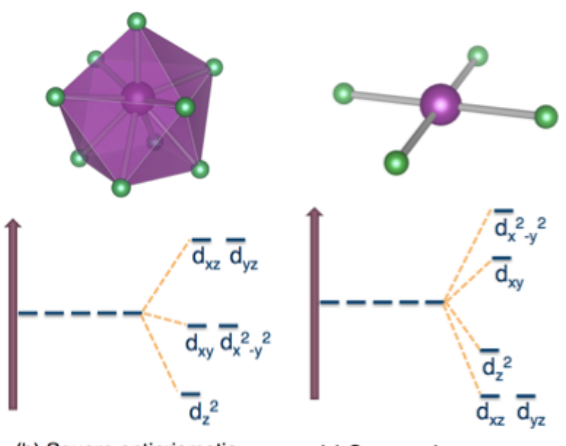

(b) Square antiprismatic

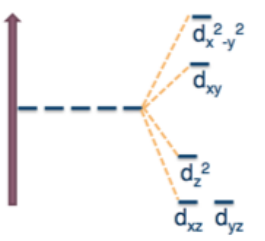

(c) Square planar
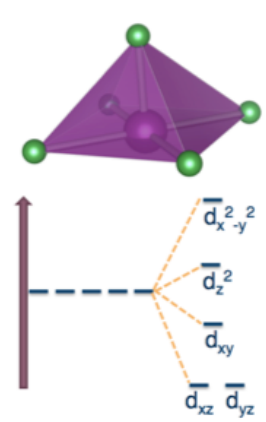

(d) Square pyramidal
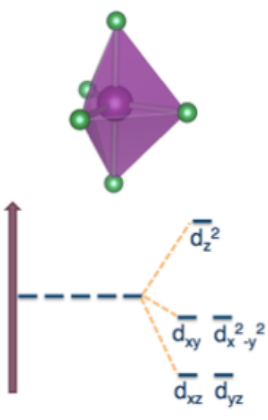

(e) Trigonal bipyramidal

FIG. 2. Common coordination environments for transition-metal ions that yield at least one singly degenerate crystal-field level. (a) pentagonal bipyramidal, (b) square antiprismatic, (c) square planar, (d) square pyramidal and (e) trigonal bipyramidal.
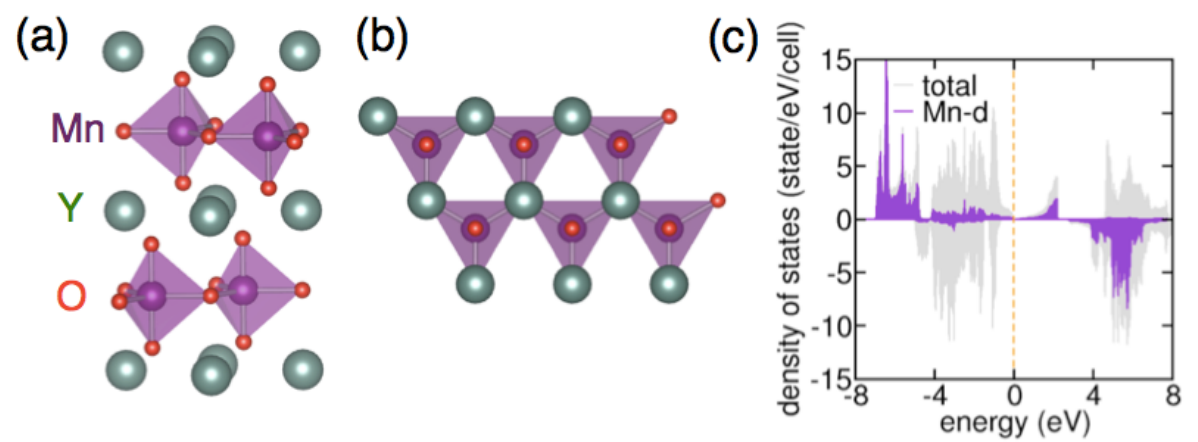

FIG. 3. (a) Side view of the hexagonal-manganite structure of $\mathrm{YMnO}_{3}$. Layers of corner-shared $\mathrm{MnO}_{5}$ trigonal bipyramids are separated by layers of Y ions. (b) Top view of the hexagonal-manganite structure showing the triangular lattice formed by the $\mathrm{Mn}$ ions which are connected via shared oxygen ions. (c) Calculated density of states of hexagonal manganite $\mathrm{YMnO}_{3}$ in its high symmetry reference structure with the Mn ions ordered ferromagnetically. The orbitally-projected Mn- $d_{z}^{2}$ states are shaded in purple and the Fermi energy, $\mathrm{E}_{F}$, is set to $0 \mathrm{eV}$. Majority (minority) spin states are shown on the positive (negative) $y$ axis. 
(a) $\mathrm{ZrCuO}_{3}$

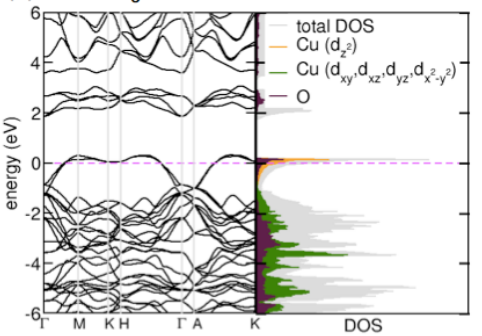

(c) $\mathrm{LiCuF}_{3}$

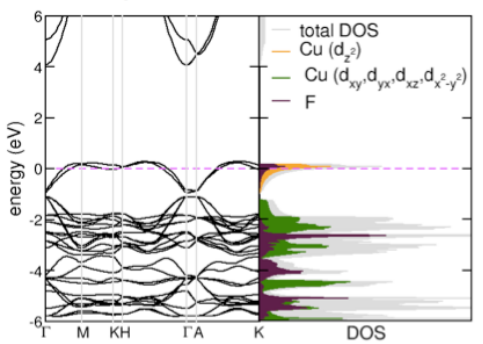

(b) $\mathrm{SnCuO}_{3}$

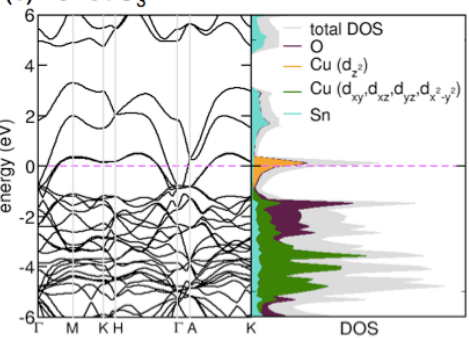

(d) $\mathrm{NaCuF}_{3}$

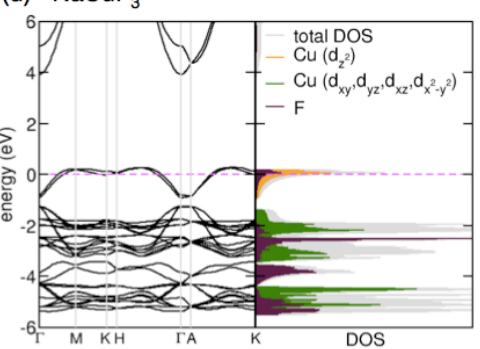

FIG. 4. Calculated band structures and total and orbital-projected densities of states (DOS) for (a) $\mathrm{ZrCuO}_{3}$, (b) $\mathrm{SnCuO}_{3}$, (c) $\mathrm{LiCuF}_{3}$ and $\mathrm{NaCuF}_{3}$, all in the hexagonal manganite structure. The Fermi level is set to $0 \mathrm{eV}$ and is marked by the dashed line in each of the plots.

(a)

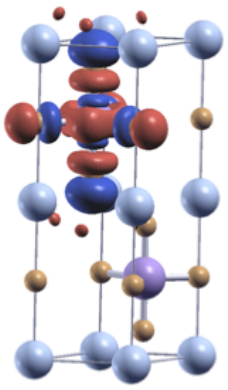

(b)

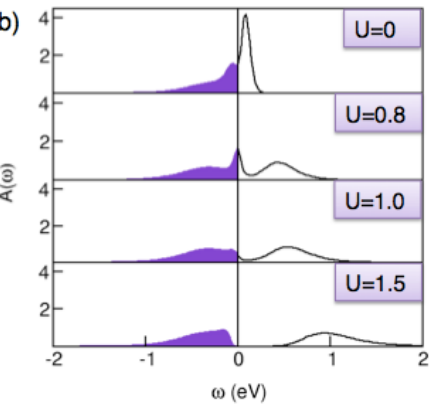

FIG. 5. (a) Maximally localized Wannier function in $\mathrm{LiCuF}_{3}$ obtained from projection of the $d_{z^{2}}$ band for one Cu site. (b) Calculated DMFT spectral function for a range of $U$ values as a function of frequency $\omega$. The Fermi energy is indicated to 0 $\mathrm{eV}$, and filled orbitals are shaded. 


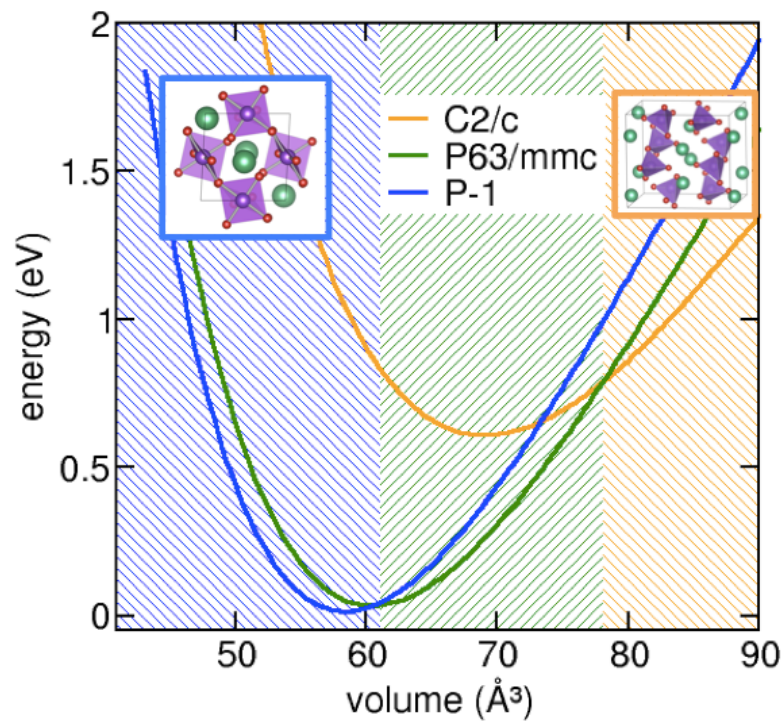

FIG. 6. Calculated total energy as a function of volume for the three calculated lowest-energy $\mathrm{LiCuF}_{3}$ isomorphs. The $\mathrm{NaCuF}_{3}$ structure (blue shaded region and inset) is the most stable structure for small volumes, and the hexagonal manganite becomes the lowest energy structure for volumes greater than $63 \AA^{3}$ (green shaded region). Finally for very large volumes the $\mathrm{CoGeO}_{3}$ structure is stabilized (shown in orange inset).
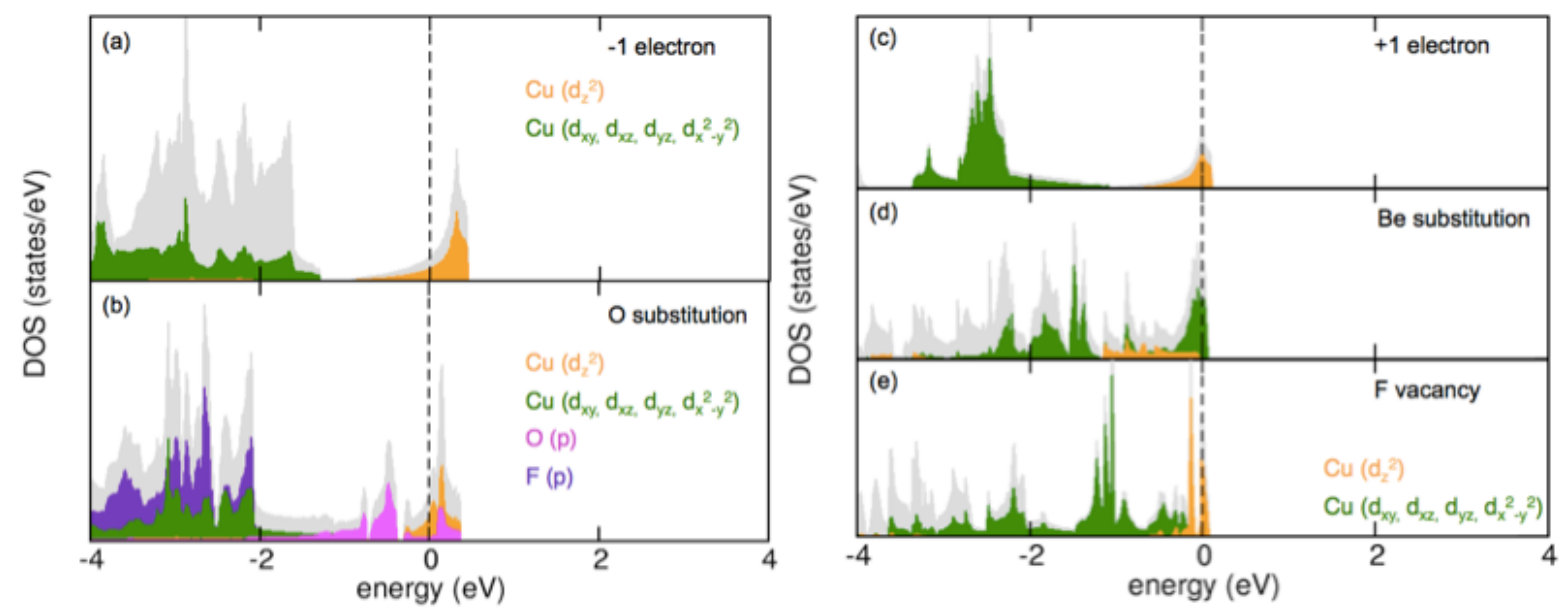

FIG. 7. Left. a) Density of states of $\mathrm{LiCuF}_{3}$ with half an electron removed per formula unit. b) Density of states for oxygensubstituted $\mathrm{Li}_{2} \mathrm{Cu}_{2} \mathrm{~F}_{5} \mathrm{O}_{1}$. Right. (c) Density of states of $\mathrm{LiCuF}_{3}$ with half an electron added per formula unit. (d) Calculated DOS for Be-substituted $\mathrm{LiCuF}_{3}$. (e) Calculated DOS for $\mathrm{LiCuF}_{3}$ with a fluorine vacancy. In all cases the Fermi level is set to $0 \mathrm{eV}$ in each case, and orbital-projected DOSs are shown for the relevant atoms. 


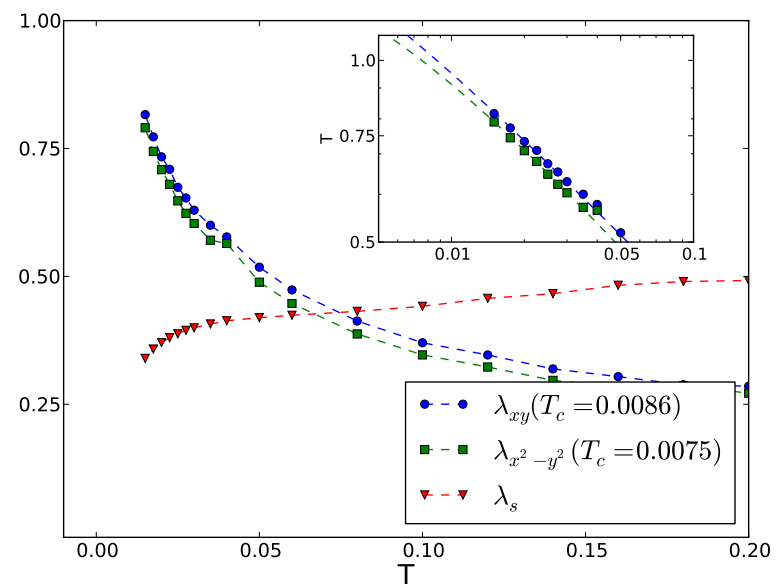

FIG. 8. The leading eigenvalue $\lambda$ of the matrix $\Gamma \chi$. A superconducting transition occurs when this leading eigenvalue $\lambda$ crosses 1 since the pairing susceptibility $\chi=\chi_{0} /(1-\Gamma \chi)$ will diverge. Inset: A log-log plot of the leading eigenvalue versus temperature. The linearity of the plot suggests that $\lambda$ behaves as $\lambda \propto \alpha\left(T-T_{c}\right)^{\gamma}$. This behaviour indicates that we are in the mean field regime. This allows us to extrapolate lambda and find a $T_{c}$ equal to $\propto 0.08$.
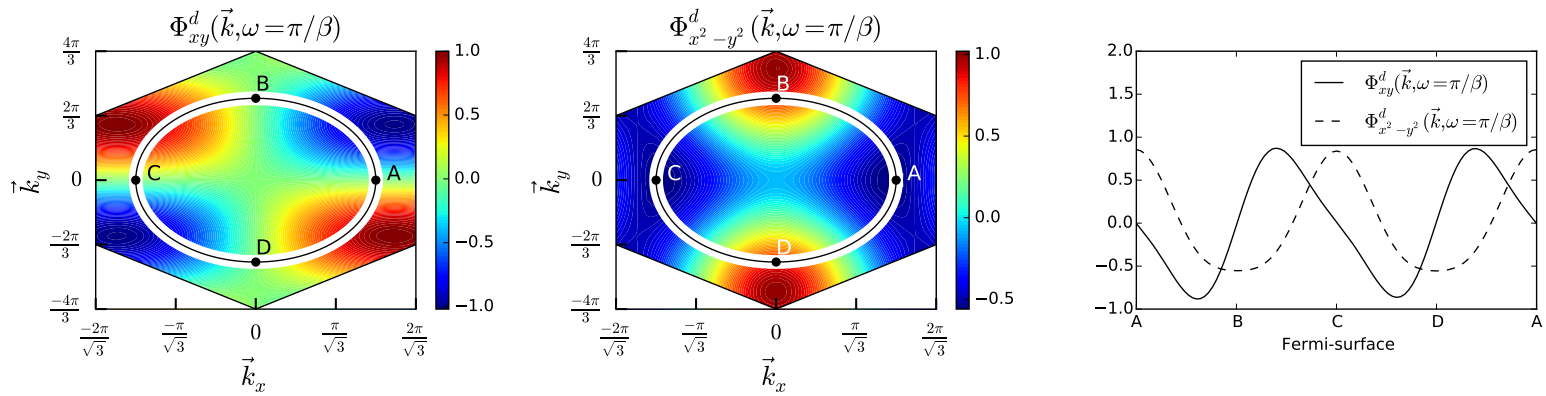

FIG. 9. The momentum space structure of the leading superconducting gap functions $\Phi^{d_{x} y}$ (left) and $\Phi^{d_{x^{2}}-y^{2}}$ (middle) at the lowest Matsubara frequency. By looking at their projections on the Fermi-surface (right), we can understand why the $\Phi^{d} x^{2}-y 2$ has a lower critical temperature. Its gap is simply not as big as the gap of $\Phi^{d_{x} y}$ in the negative region. Since the critical temperature is proportional to the size of the gap, one would expect a lower $T_{c}$ for $\Phi^{d_{x^{2}}-y^{2}}$ than for $\Phi^{d_{x} y}$. 


\begin{tabular}{|l||c|c|c|c|}
\hline & $\begin{array}{c}\text { a } \\
(\AA)\end{array}$ & $\begin{array}{c}\text { c } \\
(\AA)\end{array}$ & $\begin{array}{c}\text { Cu-F (in plane) } \\
(\AA)\end{array}$ & $\begin{array}{c}\text { Cu-F (apical) } \\
(\AA)\end{array}$ \\
\hline $\mathrm{ZrCuO}_{3}$ & 3.75 & 9.33 & 2.16 & 1.81 \\
\hline $\mathrm{SnCuO}_{3}$ & 3.78 & 9.38 & 2.18 & 1.82 \\
\hline $\mathrm{LiCuF}_{3}$ & 3.47 & 11.13 & 2.00 & 1.85 \\
\hline $\mathrm{NaCuF}_{3}$ & 3.60 & 11.60 & 2.08 & 1.84 \\
\hline $\mathrm{ZrCuS}_{3}$ & 4.69 & 10.26 & 2.70 & 2.22 \\
\hline $\mathrm{SnCuS}_{3}$ & 4.72 & 10.94 & 2.71 & 2.36 \\
\hline
\end{tabular}

TABLE I. Calculated structural parameters for hypothetical oxides, fluorides and sulphides in the centrosymmetric hexagonal manganite structure with the $P 6_{3} / m m c$ space group. The lattice parameters, $a$ and $c$ are given in the standard hexagonal setting with two formula units per unit cell. 